\title{
Investigating the discrepancy property of de Bruijn sequences
}

\author{
Daniel Gabric Joe Sawada*
}

September 9, 2021

\begin{abstract}
The discrepancy of a binary string refers to the maximum (absolute) difference between the number of ones and the number of zeroes over all possible substrings of the given binary string. We provide an investigation of the discrepancy of over a dozen simple constructions of de Bruijn sequences as well as de Bruijn sequences based on linear feedback shift registers whose feedback polynomials are primitive. Furthermore, we demonstrate constructions that attain the lower bound of $\Theta(n)$ and a new construction that attains the previously known upper bound of $\Theta\left(\frac{2^{n}}{\sqrt{n}}\right)$. This extends the work of Cooper and Heitsch [Discrete Mathematics, 310 (2010)].
\end{abstract}

\section{Introduction}

Let $\mathbf{B}(n)$ denote the set of binary strings of length $n$. A de Bruijn sequence is a circular string of length $2^{n}$ that contains every string in $\mathbf{B}(n)$ as a substring. Thus, each length- $n$ substring must occur exactly once. As an example,

$$
000000 \underline{111110111100111010111000110110100110010110000101010001001}
$$

is a de Bruijn sequence of order $n=6$; it contains each length- 6 binary string as a substring when viewed circularly. There is an extensive literature on de Bruijn sequences motivated in part by their random-like properties. As articulated by Golomb [19], de Bruijn sequences have the following properties:

- they are balanced, as they contain the same number of 0 s and $1 \mathrm{~s}$;

- they satisfy a run property, as there are an equal number of contiguous runs of 0 s and $1 \mathrm{~s}$ of the same length in the sequence;

- they satisfy a span-n property, as they contain every distinct length $n$ binary string as a substring.

From the example in (1) for $n=6$, note that there are exactly $2^{n-1} 0$ s and 1 s respectively; there are $2^{n-2}$ contiguous runs of $0 \mathrm{~s}$ and 1 s respectively; and by definition, it contains every distinct length $n$ binary string as a substring.

Despite these properties, many de Bruijn sequences display other properties that are far from random. For instance, consider the greedy prefer-1 construction [24]. After starting with an initial seed, successive bits are appended by always trying a 1 first. Only if adding a 1 results in repeating a length- $n$ substring will a 0 be appended instead. The resulting de Bruijn sequence for $n=6$ is the one obtained in (1) by rotating the

\footnotetext{
* Research supported by the Natural Sciences and Engineering Research Council of Canada (NSERC) grant RGPIN-2018-04211.
} 
initial prefix of 0 s to the suffix. As one would expect, it has more $1 \mathrm{~s}$ than $0 \mathrm{~s}$ at the start of the sequence. One measure that accounts for this is the discrepancy, which is informally defined to be the maximum absolute difference between the number of 0 s and $1 \mathrm{~s}$ in any substring of a given sequence.

To formally define discrepancy, we first introduce some notation. Let $w$ be a binary string. Let $|w|_{a}$ denote the number of occurrences of the symbol $a$ in $w$. Let $\mathbf{C}(w)$ denote the set of all substrings of $w$ where we interpret $w$ as a circular string. For example, taking $w=110$ we have that $|w|_{1}=2$ and $\mathbf{C}(w)=$ $\{\epsilon, 0,1,11,10,01,110,101,011\}$. Then the discrepancy $\operatorname{disc}(w)$ of $w$ is defined as

$$
\operatorname{disc}(w)=\left.\max _{u \in \mathbf{C}(w)}|| u\right|_{1}-|u|_{0} \mid
$$

The discrepancy of the sequence in (1) is $|17-5|=12$ as witnessed by the underlined substring. The sequences generated by the prefer- 1 construction are known to have discrepancy $\Theta\left(\frac{2^{n} \log n}{n}\right)$ [6] with an exact formulation based on the Fibonacci and Lucas numbers [7]. In contrast, the expected discrepancy of a random sequence of length $2^{n}$ is $\Theta\left(2^{n / 2} \sqrt{\log n}\right)$ [6]. Letting $\mathcal{D}$ be a de Bruijn sequence of order $n, \operatorname{disc}(\mathcal{D})$ is bounded below by $n$ since $\mathcal{D}$ must contain $1^{n}$ as a substring. In other words, $\operatorname{disc}(\mathcal{D}) \in \Omega(n)$. By putting upper bounds on character sums of non-linear recurrence sequences, an upper bound of $\operatorname{disc}(\mathcal{D}) \in O\left(2^{n} / \sqrt{n}\right)$ can be obtained; see page 1131 in [4] for an explicit calculation. One of the main results of this paper is to build on the preliminary work by the authors [16] to demonstrate de Bruijn sequence constructions with discrepancies that obtain these asymptotic lower and upper bounds.

Some applications in pseudo-random bit generation require de Bruijn sequences that do not have large discrepancy. For example, when used as a carrier signal, a de Bruijn sequence with a large discrepancy causes spectral peaks that could interfere with devices operating at these frequencies [26]. Similar measures described as "balance" and "uniformity" are discussed in [21]. However, they focus only on $n=2$ and instead vary the size of the alphabet. They explain that de Bruijn sequences with good balance and uniformity are useful in the planning of reaction time experiments [11,34]. De Bruijn sequences with high discrepancy necessarily have poor balance and uniformity.

In this paper, we extend the work initiated by Cooper and Heitsch [6] providing a more complete analysis of discrepancy for a wide variety of de Bruijn sequence constructions.

1. We evaluate the discrepancies of an additional 12 simple/interesting de Bruijn sequence constructions up to $n=30$.

2. We evaluate the discrepancies of all de Bruijn sequences obtained from linear feedback shift registers (LFSRs) based on primitive polynomials up to $n=25$.

3. We demonstrate de Bruijn sequences constructions with discrepancy that attain the asymptotic lower bound of $\Theta(n)$.

4. We present a new de Bruijn sequence construction with discrepancy that attains the asymptotic upper bound of $\Theta\left(\frac{2^{n}}{\sqrt{n}}\right)$.

The remainder of this paper is presented as follows. In Section 1.1 we present background definitions and notation. In Section 1.2 we present an overview of our experimental results. They are partitioned into five groups which are further analyzed in Sections 2, 3, 4, 5, and 6, We conclude in Section 7 with open problems and future avenues of research. 


\subsection{Background and notation}

Let $\alpha=a_{1} a_{2} \cdots a_{n}$ be a binary string in $\mathbf{B}(n)$. A feedback function is a function $f$ that maps $\mathbf{B}(n)$ to $\{0,1\}$. A feedback shift register (FSR) is a function on $\mathbf{B}(n)$ that maps a string $\alpha$ to $a_{2} a_{3} \cdots a_{n} f(\alpha)$, where $f(\alpha)$ is feedback function. If $f$ is linear, then an FSR is called a linear feedback shift register (LFSR). The following four "simple" LFSRs are presented on page 171 in the third edition of the classic work by Golomb [20]. We follow their notation letting the operator $\oplus$ represent addition modulo 2 .

- The pure cycling register (PCR) has feedback function $f(\alpha)=a_{1}$.

- The complemented cycling register (CCR) has feedback function $f(\alpha)=1 \oplus a_{1}$.

- The pure summing register (PSR) has feedback function $f(\alpha)=a_{1} \oplus a_{2} \oplus \cdots \oplus a_{n}$.

- The complemented summing register (CSR) has feedback function $f(\alpha)=1 \oplus a_{1} \oplus a_{2} \oplus \cdots \oplus a_{n}$.

In addition to these four LFSRs, there is one other LFSR that relates to the de Bruijn sequences under investigation [3,29].

- The pure run-length register (PRR) has feedback function $f(\alpha)=a_{1} \oplus a_{2} \oplus a_{n}$.

Let $0^{n}$ denote $n$ copies of 0 concatenated together. When the feedback function of an LFSR is based on a primitive polynomial (discussed further in Section 6), then its corresponding LFSR produces a maximallength sequence or m-sequence, which is a de Bruijn sequence without the $0^{n}$ substring. Adding an additional 0 before the substring $0^{n-1} 1$ in an m-sequence yields a regular de Bruijn sequence.

For the remainder of the paper, when discussing a specific algorithm for constructing a de Bruin sequence we will put it in bold, e.g., the greedy Prefer-1 construction.

\subsection{The discrepancy of de Bruijn sequence constructions up to $n=25$}

In Table 1 we present exact discrepancies for 13 de Bruijn sequence constructions for values of $n$ between 10 and 25. The results are partitioned into the following four groups based on increasing discrepancy. A larger table up to $n=30$ is provided in the appendix.

Group 1: Constructions based on the CCR.

Group 2: Constructions based on the PRR, including the greedy prefer-same (Prefer-same) and preferopposite (Prefer-opposite) constructions.

Group 3: Constructions based on the PCR, including the Prefer-1 construction. Table 1 also shows a random entry based on taking the average discrepancy of 10000 randomly generated 1 sequences of length $2^{n}$.

Group 4: Constructions based on joining smaller weight-range cycles, including one based on the PSR/CSR.

Details about the constructions from each group are presented in their respective upcoming sections. Implementations for each of these constructions can be found at http://debruijnsequence.org Each de Bruijn sequence can be generated in $O(n)$ time or better per bit using only $O(n)$ space.

\footnotetext{
${ }^{1}$ The sequences were generated in $\mathrm{C}$ using the srand and rand functions.
} 


\begin{tabular}{l|cccc|ccc} 
& \multicolumn{7}{|c}{ ( Group 1 ) } \\
$n$ & Huang & CCR2 & CCR3 & CCR1 & Pref-same & $\begin{array}{c}\text { (Group 2 ) } \\
\text { Lex-comp }\end{array}$ & Pref-opposite \\
\hline 10 & 12 & 13 & 13 & 16 & 24 & 24 & 27 \\
11 & 13 & 14 & 15 & 18 & 29 & 29 & 34 \\
12 & 15 & 16 & 16 & 22 & 35 & 35 & 43 \\
13 & 16 & 17 & 18 & 23 & 43 & 43 & 52 \\
14 & 18 & 19 & 20 & 30 & 48 & 48 & 63 \\
15 & 19 & 21 & 21 & 29 & 59 & 59 & 74 \\
16 & 21 & 22 & 23 & 36 & 68 & 68 & 87 \\
17 & 22 & 24 & 25 & 37 & 79 & 79 & 100 \\
18 & 24 & 26 & 26 & 43 & 88 & 88 & 115 \\
19 & 25 & 27 & 28 & 43 & 103 & 103 & 130 \\
20 & 27 & 29 & 30 & 52 & 114 & 114 & 147 \\
21 & 28 & 31 & 31 & 50 & 127 & 127 & 164 \\
22 & 30 & 32 & 33 & 59 & 142 & 142 & 183 \\
23 & 31 & 34 & 35 & 59 & 155 & 155 & 202 \\
24 & 33 & 36 & 36 & 67 & 172 & 172 & 223 \\
25 & 35 & 37 & 38 & 66 & 187 & 187 & 244
\end{tabular}

\begin{tabular}{|c|c|c|c|c|c|c|c|}
\hline \multirow[b]{2}{*}{$n$} & \multicolumn{5}{|c|}{ ( Group 3 ) } & \multicolumn{2}{|c|}{ ( Group 4$)$} \\
\hline & PCR4 & Random & PCR3 & PCR2 & Prefer-1/PCR1 & Cool-lex & Weight-range \\
\hline 10 & 29 & 50 & 75 & 101 & 120 & 131 & 131 \\
\hline 11 & 41 & 71 & 141 & 180 & 222 & 257 & 257 \\
\hline 12 & 51 & 101 & 248 & 321 & 416 & 468 & 468 \\
\hline 13 & 70 & 143 & 468 & 587 & 784 & 801 & 930 \\
\hline 14 & 85 & 203 & 850 & 1065 & 1488 & 1723 & 1723 \\
\hline 15 & 110 & 288 & 1604 & 1974 & 2824 & 3439 & 3439 \\
\hline 16 & 175 & 407 & 2965 & 3632 & 5376 & 6443 & 6443 \\
\hline 17 & 246 & 575 & 5594 & 6785 & 10229 & 11452 & 12878 \\
\hline 18 & 326 & 815 & 10461 & 12635 & 19484 & 24319 & 24319 \\
\hline 19 & 462 & 1157 & 19765 & 23746 & 37107 & 48629 & 48629 \\
\hline 20 & 730 & 1634 & 37243 & 44585 & 71250 & 92388 & 92388 \\
\hline 21 & 954 & 2311 & 70575 & 84270 & 138332 & 167975 & 184766 \\
\hline 22 & 1327 & 3264 & 133737 & 159281 & 268582 & 352727 & 352727 \\
\hline 23 & 1820 & 4565 & 254322 & 302449 & 521553 & 705443 & 705443 \\
\hline 24 & 2684 & 6252 & 484172 & 574819 & 1012795 & 1352090 & 1352090 \\
\hline 25 & 3183 & 9192 & 924071 & 1096009 & 1966813 & 2496163 & 2704168 \\
\hline
\end{tabular}

Table 1: Discrepancies of de Bruijn sequence constructions of order $n$ ordered by increasing discrepancy and partitioned into four groups.

We also consider a fifth group of de Bruijn sequences, where each sequence corresponds to a unique primitive polynomial.

Group 5: Constructions based on primitive polynomials and their corresponding LFSRs.

By generating all primitive polynomials of degree $n$ and their corresponding LFSRs, we compute the minimum, the maximum, and average discrepancies for their corresponding de Bruijn sequences in Table 2, Any related $\mathrm{m}$-sequence known to be generated by such an LFSR can be completely determined after reading only $2 n$ bits [25]. Thus, their application for generating pseudo-random numbers is limited. Further discussion is given in Section 6

\subsection{Computing the discrepancy of a de Bruijn sequence}

Given a de Bruijn sequence of order $n$, how quickly can the discrepancy be calculated? De Bruijn sequences are exponentially long with respect to their order. Thus, it is natural to try to find a fast algorithm to compute the discrepancy of de Bruijn sequences. 


\begin{tabular}{r|rrrr|r} 
& \multicolumn{6}{|c}{ ( Group 5 ) } & \\
$n$ & min & avg & Random & max & LFSRs \\
\hline 10 & 36 & 41 & 50 & 46 & 60 \\
11 & 51 & 58 & 71 & 68 & 176 \\
12 & 72 & 84 & 101 & 106 & 144 \\
13 & 97 & 118 & 143 & 144 & 630 \\
14 & 141 & 167 & 203 & 206 & 756 \\
15 & 200 & 236 & 288 & 294 & 1800 \\
16 & 280 & 335 & 407 & 432 & 2048 \\
17 & 391 & 473 & 575 & 625 & 7710 \\
18 & 544 & 669 & 815 & 860 & 7776 \\
19 & 775 & 947 & 1157 & 1262 & 27594 \\
20 & 1066 & 1341 & 1634 & 1842 & 24000 \\
21 & 1500 & 1896 & 2311 & 2619 & 84672 \\
22 & 2128 & 2681 & 3264 & 3634 & 120032 \\
23 & 3009 & 3793 & 4565 & 5326 & 356960 \\
24 & 4236 & 5362 & 6252 & 7545 & 276480 \\
25 & 5905 & 7586 & 9192 & 11291 & 1296000
\end{tabular}

Table 2: The minimum, average, and maximum discrepancies of de Bruijn sequence constructions of order $n$ based on primitive polynomials and their corresponding LFSRs. The number of LFSRs based on primitive polynomials is given in the final column.

A naïve way to calculate the discrepancy of a de Bruijn sequence $\mathcal{D}$ is to consider every substring $u$ of $\mathcal{D}$ and compute $\left.|| u\right|_{1}-|u|_{0} \mid$, keeping track of the maximum value. In a circular string of length $m$, there are $m$ substrings of length $1, m$ substrings of length 2 , and more generally $m$ substrings of length $j$. Therefore, there are $\Theta\left(m^{2}\right)$ substrings in a length- $m$ circular string. For every substring $u$ that the algorithm visits, the absolute difference between the number of 0 s and the number of 1 s in $u$ is computed, which takes $\Theta(|u|)$ time. Thus, when given a de Bruijn sequence of order $n$ as input, this algorithm runs in $\Theta\left(2^{3 n}\right)$ time. A slight upgrade from this naïve approach is obtained by observing that every substring of a circular string $w$ is a prefix of a rotation of $w$. For every rotation of $w$, we scan from left to right one bit at a time while keeping track of the number of $0 \mathrm{~s}$, the number of $1 \mathrm{~s}$, and the maximum absolute difference between them. This algorithm runs in $\Theta\left(2^{2 n}\right)$ time when given a de Bruijn sequence of order $n$ as input.

We show that the discrepancy of a de Bruijn sequence of order $n$ can be calculated in $\Theta\left(2^{n}\right)$ time. First, we define some notation. Let $w$ be a binary string and let

$$
d_{0}(w)=\max _{w=u v}\left(|u|_{0}-|u|_{1}\right)
$$

and

$$
d_{1}(w)=\max _{w=u v}\left(|u|_{1}-|u|_{0}\right) .
$$

In other words, $d_{0}(w)$ (resp., $d_{1}(w)$ ) denotes the maximum difference between the numbers of 0 s and $1 \mathrm{~s}$ (resp., 1s and 0s) in any prefix of $w$.

Lemma 1.1 Let $\mathcal{D}$ be a de Bruijn sequence of order $n$. There exists a string $y$ such that $\mathcal{D}=x y z$ and $\operatorname{disc}(\mathcal{D})=\left.|| y\right|_{1}-|y|_{0} \mid$.

Proof. Suppose we cannot write $\mathcal{D}=x y z$ where $\operatorname{disc}(\mathcal{D})=\left.|| y\right|_{1}-|y|_{0} \mid$ for any strings $x, y, z$. Then we must have $\operatorname{disc}(\mathcal{D})=\left.|| z x\right|_{1}-|z x|_{0} \mid$ for some choice of $x, z$. However, since $\mathcal{D}$ is a de Bruijn sequence, it must contain the same number of 1 s as 0 s. $\operatorname{Thus,} \operatorname{disc}(\mathcal{D})=\left.|| z x\right|_{1}-|z x|_{0}|=||y|_{1}-|y|_{0} \mid$, which contradicts our initial assumption. 
Lemma 1.2 Let $\mathcal{D}$ be a de Bruijn sequence of order $n$. Then $\operatorname{disc}(\mathcal{D})=d_{0}(\mathcal{D})+d_{1}(\mathcal{D})$.

Proof. Let $\gamma_{i}$ denote the length- $i$ prefix of $\mathcal{D}$. Let $j_{0}, j_{1}, \ldots, j_{2^{n}}$ denote a sequence of integers such that $j_{0}=0$ and $j_{i}=\left|\gamma_{i}\right|_{1}-\left|\gamma_{i}\right|_{0}$ for all $i \in\left\{1,2, \ldots, 2^{n}\right\}$. Since $\mathcal{D}$ is a de Bruijn sequence, it has an equal number of $1 \mathrm{~s}$ and 0s. Thus, $j_{2^{n}}=0$. By Lemma 1.1, we can write $\mathcal{D}=x y z$ for strings $x, y, z$ such that $\operatorname{disc}(\mathcal{D})=\left.|| y\right|_{1}-|y|_{0} \mid$. The number of $1 \mathrm{~s}$ in $y$ is equal to the number of $1 \mathrm{~s}$ in $x y$ minus the number of $1 \mathrm{~s}$ in $x$. The same is true for the number of $0 \mathrm{~s}$ in $y$. Therefore, $|y|_{1}-|y|_{0}=j_{|x y|}-j_{|x|}$. The value $\left|j_{|x y|}-j_{|x|}\right|$ is maximized when either $j_{|x|}$ is as large as possible and $j_{|x y|}$ is as small as possible, or when $j_{|x|}$ is as small as possible and $j_{|x y|}$ is as large as possible. In the former case, the value corresponds to $d_{1}(\mathcal{D})-\left(-d_{0}(\mathcal{D})\right)$, and in the latter case the value corresponds to $\left|-d_{0}(\mathcal{D})-d_{1}(\mathcal{D})\right|$. In both cases the value simplifies to $d_{1}(\mathcal{D})+d_{0}(\mathcal{D})$.

Corollary 1.3 Let $\mathcal{D}$ be a de Bruijn sequence of order $n$. The discrepancy of $\mathcal{D}$ can be calculated in $\Theta(|\mathcal{D}|)$ time.

\section{Group 1: CCR-based constructions}

In this section we consider the four de Bruijn sequence constructions in Group 1 based on the CCR. The sequences generated by the constructions CCR1, CCR2, and CCR3 are based on shift-rules presented in [18]. The sequences generated by the CCR2 and CCR3 constructions can also be constructed by concatenation approaches [17] described later in this section; the equivalence of the shift-rules to their respective concatenation constructions has been confirmed up to $n=30$, though no formal proof has been given. The Huang construction is a shift-rule based construction in [22]. Since every de Bruijn sequence of order $n$ contains the substring $0^{n}$, a lower bound on discrepancy is clearly $n$. In this section we prove that two aforementioned concatenation based constructions have discrepancy at most $2 n$, and thus attain the smallest possible asymptotic discrepancy of $\Theta(n)$.

To get a better feel for these four de Bruijn sequence constructions, the following graphs illustrate the running difference between the number of $1 \mathrm{~s}$ and the number of $0 \mathrm{~s}$ in each prefix of the given de Bruijn sequence. The examples are for $n=10$, so the de Bruijn sequences have length $2^{10}=1024$.
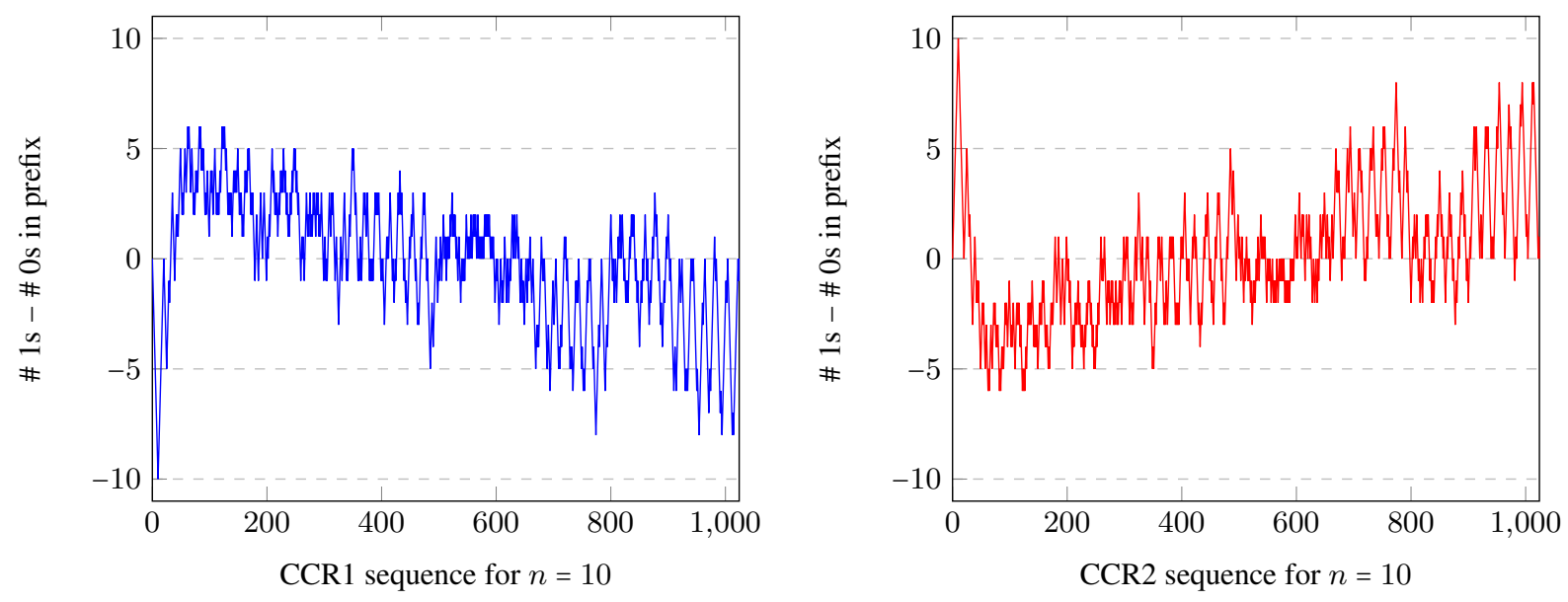

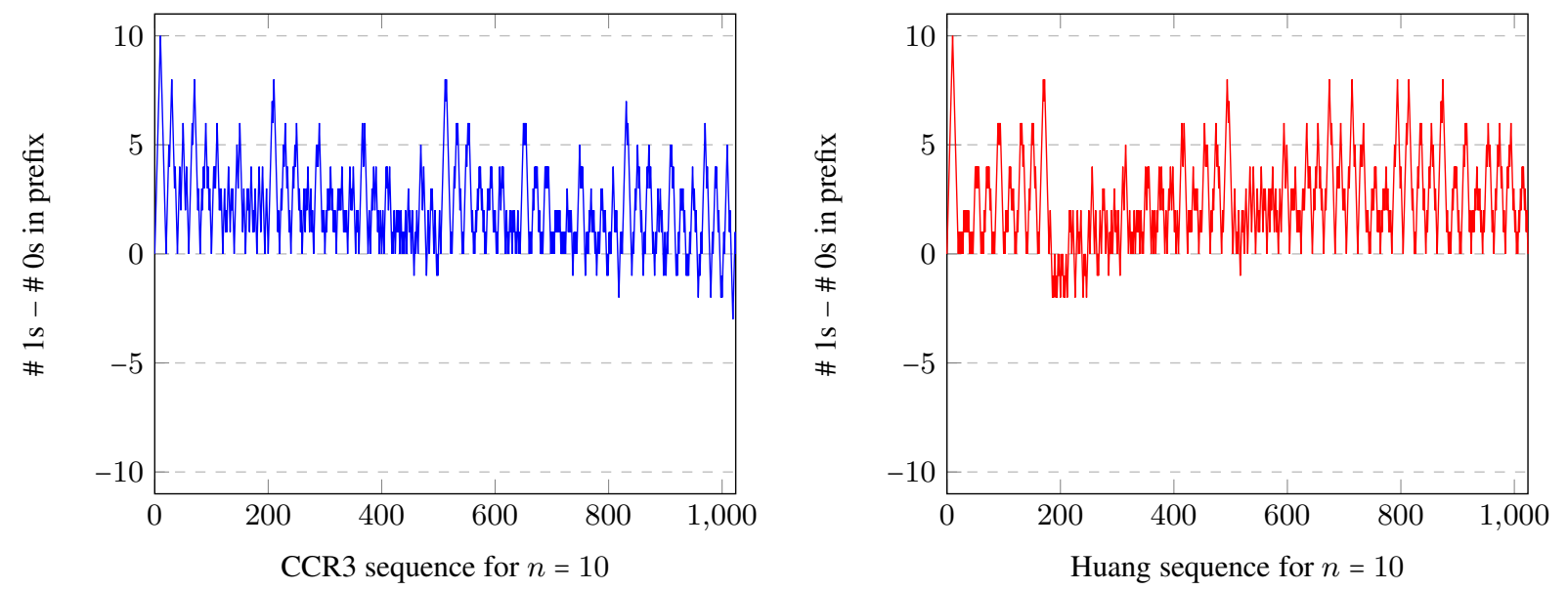

Recall that the CCR is a feedback shift register with feedback function $f\left(a_{1} a_{2} \cdots a_{n}\right)=a_{1}+1(\bmod 2)$. $\mathbf{B}(n)$ is partitioned into equivalence classes of strings, called co-necklaces, by the orbits of $f$. For example, the following four columns are the co-necklace equivalence classes for $n=5$ :

$\begin{array}{llll}\mathbf{0 0 0 0 0} & \mathbf{0 0 0 1 0} & \mathbf{0 0 1 0 0} & \mathbf{0 1 0 1 0} \\ 00001 & 00101 & 01001 & \underline{10101} \\ 00011 & 01011 & \underline{10011} & \\ 00111 & \underline{10111} & 00110 & \\ 01111 & 01110 & 01101 & \\ \underline{11111} & 11101 & 11011 & \\ 11110 & 11010 & 10110 & \\ 11100 & 10100 & 01100 & \\ 11000 & 01000 & 11001 & \\ 10000 & 10001 & 10010 & \end{array}$

The periodic reduction of a string $\alpha$, denoted by $\operatorname{pr}(\alpha)$, is the shortest prefix $\beta$ of $\alpha$ such that $\alpha=\beta^{j}$ for some $j \geq 1$. In [17], the following two de Bruijn sequence constructions CCR2 and CCR3 concatenate the periodic reductions of $\alpha \bar{\alpha}$ for given representatives $\alpha$ of each co-necklace equivalence class.

\section{CCR2}

1. Let the representative for each co-necklace equivalence class of order $n$ be its lexicographically smallest string.

2. Let $\alpha_{1}, \alpha_{2}, \ldots, \alpha_{m}$ denote these representatives in colex order.

3. Output: $\operatorname{pr}\left(\alpha_{1} \overline{\alpha_{1}}\right) \cdot \operatorname{pr}\left(\alpha_{2} \overline{\alpha_{2}}\right) \cdots \operatorname{pr}\left(\alpha_{m} \overline{\alpha_{m}}\right)$.

For $n=5$, the representatives for this algorithm are the bolded strings in the equivalence classes above and CCR2 produces:

$$
0000011111 \cdot 0010011011 \cdot 0001011101 \cdot 01
$$




\section{CCR3}

1. Let the representative for each co-necklace equivalence class of order $n$ be the string obtained by taking the lexicographically smallest string, removing its largest prefix of the form $0^{j}$, and then appending $1^{j}$ to the end.

2. Let $\alpha_{1}, \alpha_{2}, \ldots, \alpha_{m}$ denote these representatives in lexicographic order.

3. Output: $\operatorname{pr}\left(\alpha_{1} \overline{\alpha_{1}}\right) \cdot \operatorname{pr}\left(\alpha_{2} \overline{\alpha_{2}}\right) \cdots \operatorname{pr}\left(\alpha_{m} \overline{\alpha_{m}}\right)$.

For $n=5$, the representatives for this algorithm are the underlined strings in the equivalence classes above and CCR3 produces:

$$
1001101100 \cdot 10 \cdot 1011101000 \cdot 1111100000 .
$$

We now prove that the discrepancy resulting from these two de Bruijn sequence constructions is at most $2 n$.

Lemma 2.1 Consider a sequence of binary strings $\alpha_{1}, \alpha_{2}, \ldots, \alpha_{m}$ where each $\alpha_{i}$ has the same number of $0 s$ as 1 s and has discrepancy at most $n$. Then $\operatorname{disc}\left(\alpha_{1} \alpha_{2} \cdots \alpha_{m}\right) \leq 2 n$.

Proof. Let $\mathcal{S}=\alpha_{1} \alpha_{2} \cdots \alpha_{m}$. By Lemma 1.1 there exists a shortest substring $y=u \alpha_{i+1} \alpha_{i+2} \cdots \alpha_{j-1} v$ of $\mathcal{S}$ such that $\operatorname{disc}(\mathcal{S})=\left.|| y\right|_{1}-|y|_{0} \mid$ where $u$ is a suffix of $\alpha_{i}$ and $v$ is a prefix of $\alpha_{j}$. Since the number of 0 s and 1 s is the same in each $\alpha_{k}$ and $\operatorname{disc}\left(\alpha_{k}\right) \leq n,\left.|| y\right|_{1}-|y|_{0}|=||u v|_{1}-|u v|_{0} \mid \leq 2 n$.

\section{Theorem 2.2 The de Bruijn sequences constructed by CCR2 and CCR3 have discrepancy at most $2 n$.}

Proof. Given a length $n$ binary string $\alpha, \alpha \bar{\alpha}$ has the same number of 0 s and 1 s and has discrepancy at most $n$. These properties also hold for $\operatorname{pr}(\alpha \bar{\alpha})$ by definition of the periodic reduction. Thus, by Lemma 2.1, the sequences constructed by CCR2 and CCR3 have discrepancy at most $2 n$.

Interestingly, from Table 1, these two concatenation-based constructions do not demonstrate the smallest discrepancy for $n \leq 30$. The construction by Huang [22], which is based on a cycle-joining approach, demonstrates slightly smaller discrepancy. In particular the author states:

"It seems clear that the sequences produced by our algorithm have a relatively good characteristic of local 0-1 balance in comparison with the ones produced by the 'prefer one' algorithm."

So the author indicates that their construction may have small discrepancy, however no analysis is provided.

\section{Group 2: PRR-based constructions}

In this section we consider the three de Bruijn sequence constructions in Group 2 based on the PRR. The Pref-same [3, 10, 13] and the Pref-opposite [2] are greedy constructions based on the last bit of the sequence as it is constructed. They have the downside of requiring an exponential amount of memory. The Lexcomp construction is obtained by concatenating lexicographic compositions. It was an attempt to generate 
the sequence generated by the Pref-same construction without using exponential space and it the resulting sequences were conjectured to be the same for a very long prefix [14]. In fact, it attains the same discrepancy as the Pref-same for all values of $n$ tested. Recently, it was demonstrated that the Pref-same and the Prefopposite sequences can be generated in $O(n)$ time per bit using only $O(n)$ space by applying the PRR [29]. There is also a PRR-based construction that produces an equivalent sequence as Lex-comp for large $n$, but there is no formal proof showing they are equivalent.

To get a better feel for the two greedy de Bruijn sequence constructions, the following graphs illustrate the running difference between the number of $1 \mathrm{~s}$ and the number of $0 \mathrm{~s}$ in each prefix of the given de Bruijn sequence. The examples are for $n=10$, so the de Bruijn sequences have length $2^{10}=1024$.
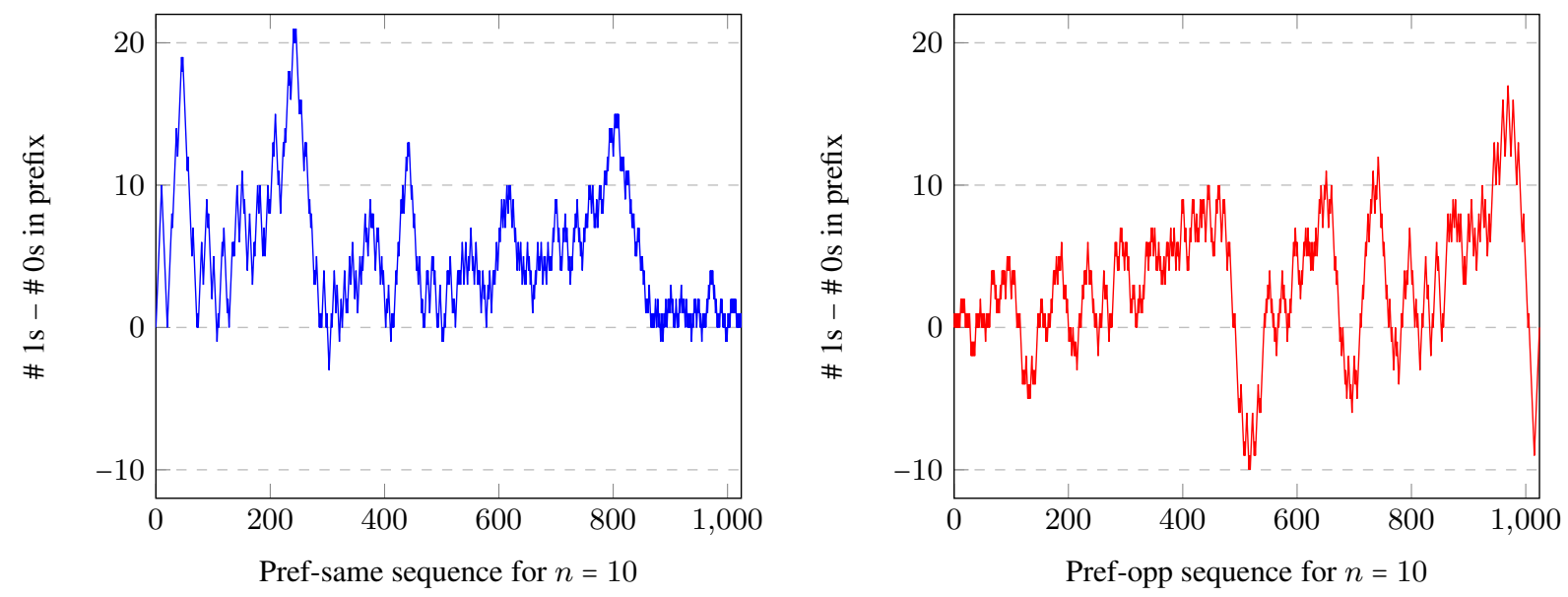

In the following table we study some experimental results for the Pref-same construction. In particular, for $10 \leq n \leq 25$ we compute the maximum difference between the number of 1 s and the number of 0 s along with the maximum difference between the number 0s and the number of $1 \mathrm{~s}$, over all prefixes of each Prefsame de Bruijn sequence of order $n$. Adding these two values together, we get the discrepancies shown in Table 1.

\begin{tabular}{c|rrrrrrrrrrrrrrrr}
$n$ & 10 & 11 & 12 & 13 & 14 & 15 & 16 & 17 & 18 & 19 & 20 & 21 & 22 & 23 & 24 & 25 \\
\hline $\max (\# 1 \mathrm{~s}-\# 0 \mathrm{~s})$ & 21 & 26 & 31 & 36 & 43 & 50 & 57 & 64 & 73 & 82 & 91 & 100 & 111 & 122 & 133 & 144 \\
$\max (\# 0 \mathrm{~s}-\# 1 \mathrm{~s})$ & 3 & 3 & 4 & 7 & 5 & 9 & 11 & 15 & 15 & 21 & 23 & 27 & 31 & 33 & 39 & 43 \\
\hline discrepancy & 24 & 29 & 35 & 43 & 48 & 59 & 68 & 79 & 88 & 103 & 114 & 127 & 142 & 155 & 172 & 187
\end{tabular}

Interestingly, the values in the row $\max (\# 1 \mathrm{~s}-\# 0$ s) are equivalent to the known sequence A008811 in the Online Encyclopedia of Integer Sequences (OEIS) [1] offset by four positions. The sequence enumerates the "Expansion of $x\left(1+x^{4}\right) /\left((1-x)^{2}\left(1-x^{4}\right)\right)$ " and the provided formula demonstrates that each value is $\Theta\left(n^{2}\right)$. More specifically the values match the sequence for $6 \leq n \leq 30$, though we have no intuition as to why this is the case. This leads to the following conjecture.

Conjecture 3.1 The de Bruijn sequences constructed by the Pref-same and Lex-comp algorithms have discrepancy $\Theta\left(n^{2}\right)$.

A similar analysis was performed for sequences generated by the Pref-opposite construction.

\begin{tabular}{c|rrrrrrrrrrrrrrrr}
$n$ & 10 & 11 & 12 & 13 & 14 & 15 & 16 & 17 & 18 & 19 & 20 & 21 & 22 & 23 & 24 & 25 \\
\hline $\max (\# 1 \mathrm{~s}-\# 0 \mathrm{~s})$ & 10 & 13 & 17 & 21 & 26 & 31 & 37 & 43 & 50 & 57 & 65 & 73 & 82 & 91 & 101 & 111 \\
$\max \# 0 \mathrm{~s}-\# 1 \mathrm{~s})$ & 17 & 21 & 26 & 31 & 37 & 43 & 50 & 57 & 65 & 73 & 82 & 91 & 101 & 111 & 122 & 133 \\
\hline discrepancy & 27 & 34 & 43 & 52 & 63 & 74 & 87 & 100 & 115 & 130 & 147 & 164 & 183 & 202 & 223 & 244
\end{tabular}


Remarkably, observe that the two middle rows are a shift from each other by two positions. Just as interesting, the sequences also correspond to a known sequence in OEIS [1], namely A033638. Specifically, the row $\max (\# 1 \mathrm{~s}-\# 0 \mathrm{~s})$ corresponds to this sequence shifted by four positions. The sequence does not match for $n<10$, but we have verified it matches for $10 \leq n \leq 30$. The sequence corresponds to "quarter squares plus 1 ", and by applying the appropriate shifts, the discrepancy for the Prefer-opposite sequence of order $n$, for $10 \leq n \leq 30$ is given by:

$$
\left\lfloor\frac{(n-4)^{2}}{4}\right\rfloor+\left\lfloor\frac{(n-2)^{2}}{4}\right\rfloor+2 .
$$

This leads to the following conjecture.

Conjecture 3.2 The de Bruijn sequence constructed by the Pref-opposite algorithm has discrepancy $\Theta\left(n^{2}\right)$.

We conclude this section with an observation regarding the Pref-opposite de Bruijn sequence: For $2 \leq$ $n \leq 25$, each sequence has the following suffix where $j=\lceil n / 3\rceil$ :

$$
0^{j} 1^{n-j} \cdot 0^{j-1} 1^{n-j+1} \cdots 01^{n-1} \cdot 10^{n-1} .
$$

For example, when $n=10$, the Pref-opposite de Bruijn sequence has suffix

$$
0000001111 \cdot 00000 \underline{11111} \cdot 0000111111 \cdot 0001111111 \cdot 0011111111 \cdot 0111111111 \cdot 1000000000,
$$

and the underlined substring has $5+6+7+8+10$ ones and $4+3+2+1$ zeros. A slight rearrangement gives a lower bound of $(5-1)+(6-2)+(7-3)+(8-4)+10=4 \cdot 4+10=26$ for the discrepancy of the sequence. The actual discrepancy is 27. More generally, if this suffix is indeed a suffix for each Pref-opposite de Bruijn sequence, then a lower bound on its discrepancy will be

$$
(\lceil n / 2\rceil-1)(\lfloor n / 2\rfloor-1)+n=\Omega\left(n^{2}\right) .
$$

\section{Group 3: PCR-based constructions}

In this section we consider the four de Bruijn sequence constructions in Group 3 based on the PCR. The constructions PCR1, PCR2, PCR3, and PCR4 are based on shift-rules presented in [18]. Like the other shift-rule constructions, these four rules result from joining smaller cycles based on the underlying feedback function; depending on how the "bridge states" are defined leads to the different shift-rules. The sequences generated by PCR1 are the same as the ones generated by the prefer- 0 greedy construction; they are the complements of the sequences generated by PCR1, and so they have the same. The sequences generated by PCR1 can also be generated by two necklace concatenation constructions, one based on lexicographic order [15], and another taking a recursive approach [27].

The sequences generated by PCR2 are the same as the ones generated by a necklace concatenation construction based on colex order [8,9]. The PCR3 is based on a general approach in [23] and revisited in [33].

To get a better feel for these four de Bruijn sequence constructions, the following graphs illustrate the running difference between the number of $1 \mathrm{~s}$ and the number of 0 s in each prefix of the given de Bruijn sequence. The examples are for $n=10$, so the de Bruijn sequences have length $2^{10}=1024$. 

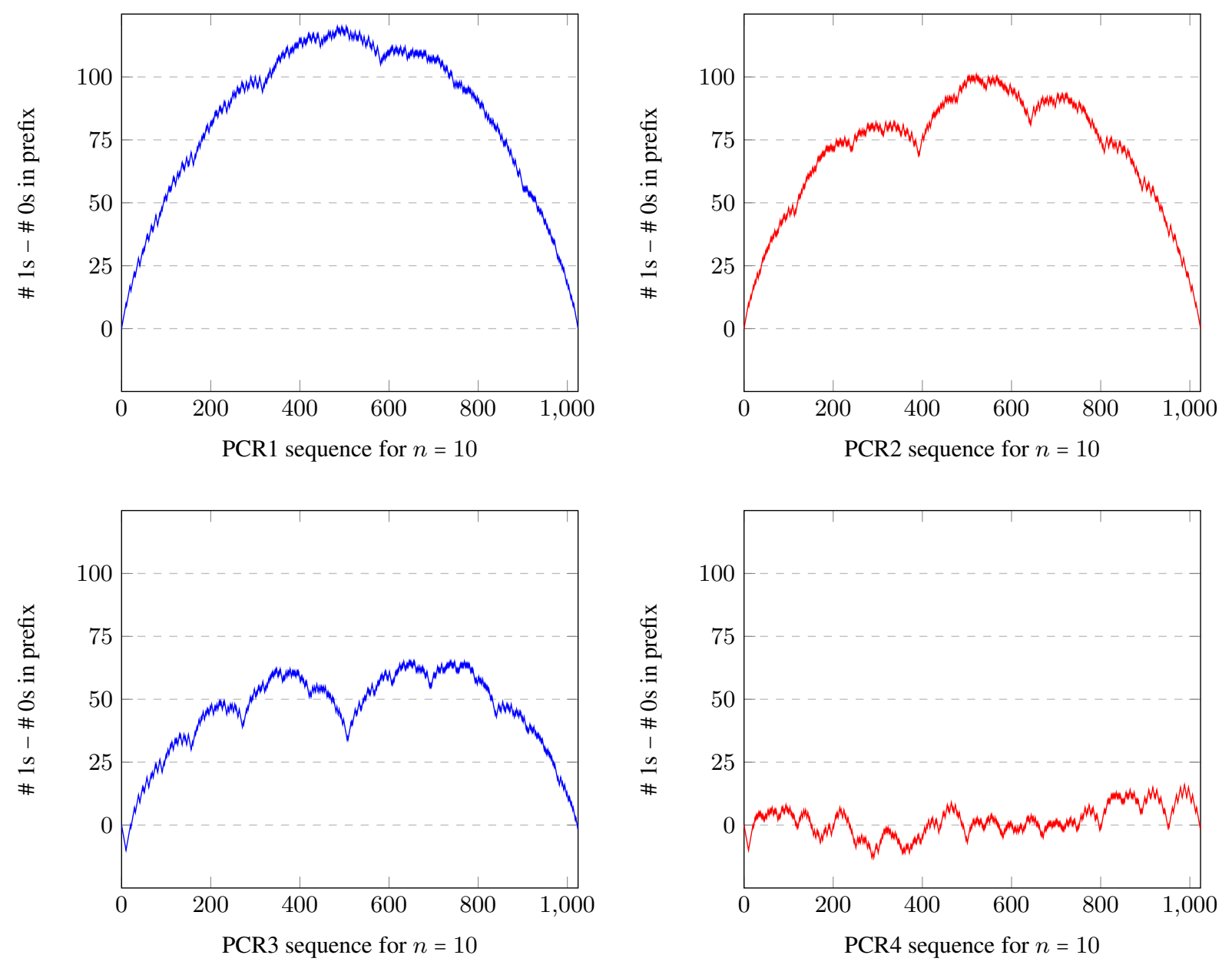

The discrepancy for the sequence generated by the PCR1 construction has already been studied in [6] where they show that the discrepancy is $\Theta\left(\frac{2^{n} \log n}{n}\right)$. The sequences generated by the PCR2 and PCR3 constructions appear to have a similar growth trajectories. More interesting are the sequences generated by the PCR4 construction that, from Table 1, appear to have discrepancy that is closest to that of a random string. It would be interesting to do a more detailed investigation of this construction, which is based on a very simple successor rule.

\section{Group 4: Weight range constructions and the PSR/CSR}

In this section we consider two de Bruijn sequence constructions that join smaller cycles based on weight (number of 1s). In some related works the term density is also used to mean weight, so will use the variable $d$ to indicate a weight. The Cool-lex construction [28], is a concatenation approach which is based on creating underlying cycles which contain all strings with weights $d$ and $d+1$ given $0 \leq d<n$. Then, appropriate such cycles can be joined together to obtain a de Bruijn sequence [31]. By the nature of how the cycles are joined, most length- $n$ substrings in the first half of the resulting de Bruijn sequence have weight less than or equal to $n / 2$. Similarly, most length- $n$ substrings in the latter half of the sequence have weight greater than or equal to $n / 2$. Thus, as one would expect, the resulting de Bruijn sequence has a very large discrepancy. The Weight-range construction is a new construction presented in this section. Its resulting de Bruijn sequence has discrepancy that attains the asymptotic upper bound of $\Theta\left(2^{n} / \sqrt{n}\right)$. 
To get a better feel for these two de Bruijn sequence constructions, the following graphs illustrate the running difference between the number of $1 \mathrm{~s}$ and the number of $0 \mathrm{~s}$ in each prefix of the given de Bruijn sequence. The examples are for $n=10$, so the de Bruijn sequences have length $2^{10}=1024$.
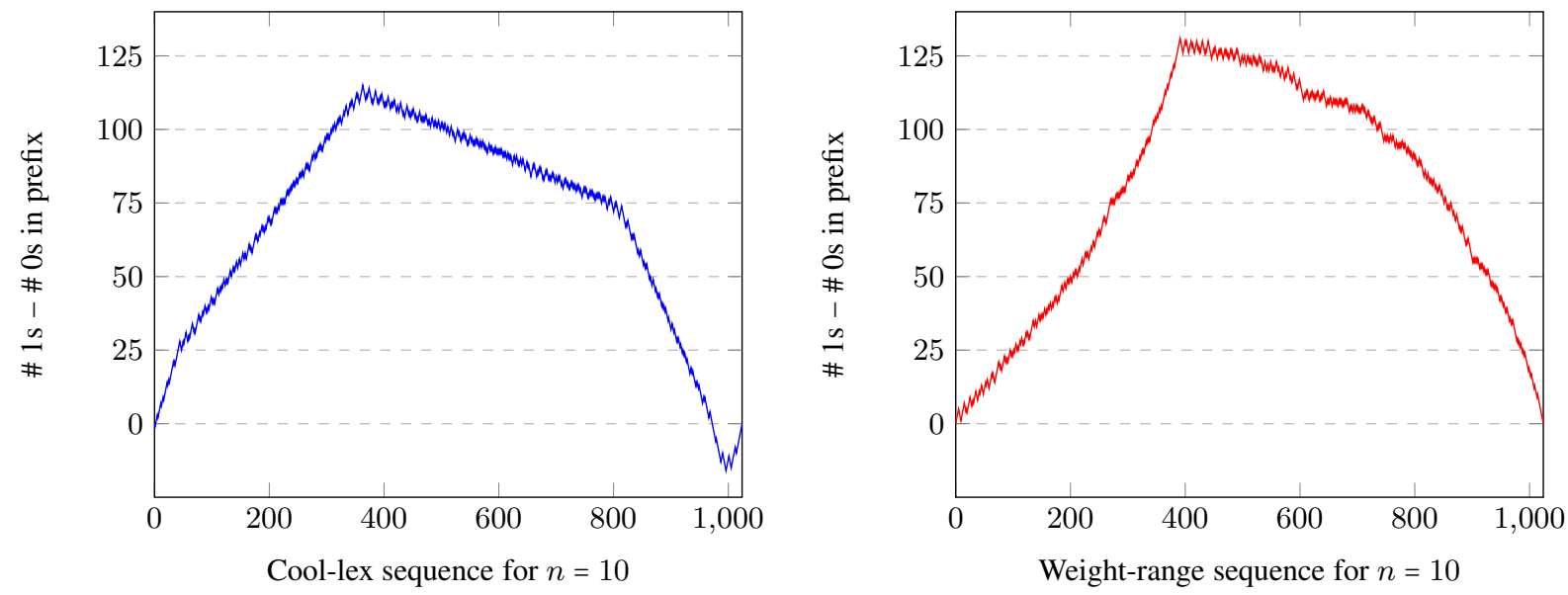

Notice that if we had shifted the starting position of the Cool-lex sequence the profile of the graph would be very similar to that the Weight-range sequence. In fact, the discrepancies of the two sequences are the same except when $n \bmod 4 \equiv 1$ (see Table 1 ). This will be discussed more after we present the Weight-range construction.

A minimum weight de Bruijn sequence is a cyclic sequence that contains each binary string of length $n$ with weight at least $d$ exactly once. A maximum weight de Bruijn sequence is defined similarly where the weight of each string is at most $d$. A construction for the former sequence is given in [32]; it is constructed by concatenating the periodic reduction of each necklace of weight $\geq d$ when the necklaces are listed in lexicographic order. Let the resulting sequence be denoted by $\mathcal{D}_{d}(n)$.

Remark 5.1 For any $d<n, \mathcal{D}_{d}(n)$ begins with $0^{n-d} 1^{d}$ and ends with $1^{n}$.

By complementing the bits in $\mathcal{D}_{d}(n)$, we obtain a maximum weight de Bruijn sequence with weight at most $n-d$. Denote this sequence by $\overline{\mathcal{D}}_{d}(n)$. From the previous remark, it begins with $1^{n-d} 0^{d}$ and ends with $0^{n}$.

Example 1 The necklaces of length 6 with weight $d \geq 3$ in lexicographic order are:

$$
\text { 000111, 001011, 001101, 001111,010101,010111,011011,011111, } 111111 .
$$

Concatenating together their periodic reductions we obtain the minimum weight de Bruijn sequence $\mathcal{D}_{3}(6)$.

$$
000111 \cdot 001011 \cdot 001101 \cdot 001111 \cdot 01 \cdot 010111 \cdot 011 \cdot 011111 \cdot 1
$$

As further examples,

$$
\mathcal{D}_{4}(6)=001111 \cdot 010111 \cdot 011 \cdot 011111 \cdot 1
$$

and

$$
\overline{\mathcal{D}}_{4}(6)=110000 \cdot 101000 \cdot 100 \cdot 100000 \cdot 0 .
$$

From the above example observe that: 
- $\mathcal{D}_{3}(6)$ contains all binary strings of length 6 with weight greater than or equal to 3 ,

- $\overline{\mathcal{D}}_{4}(6)$ contains all binary strings of length 6 with weight less than or equal to 2 ,

- The length $n-1$ prefix of $\overline{\mathcal{D}}_{4}(6)$, namely 11000 , appears in the wraparound of $\mathcal{D}_{3}(6)$.

Let $\mathcal{D}_{d}^{r}(n)$ denote the sequence $\mathcal{D}_{d}(n)$ with the suffix $1^{d-1}$ rotated to the front. Then by applying the Gluing Lemma [31], the following is a de Bruijn sequence of order 6 :

$$
\begin{aligned}
& \underline{1100001010001001000000} \cdot \underline{110001110010110011010011110101011101101111 .} \\
& \overline{\mathcal{D}}_{4}(6)
\end{aligned}
$$

Applying this strategy more generally, let $\mathcal{D} \mathcal{B}_{\max }(n)$ denote the de Bruijn sequence obtained by joining two such smaller cycles.

\section{Weight-range construction}

$$
\mathcal{D} \mathcal{B}_{\text {max }}(n)=\overline{\mathcal{D}}_{d}(n) \cdot \mathcal{D}_{d^{\prime}}^{r}(n)
$$

where $d=\lfloor n / 2\rfloor+1$ and $d^{\prime}=\lceil n / 2\rceil$.

A complete $\mathrm{C}$ implementation to construct $\mathcal{D B}_{\text {max }}(n)$ is given in the Appendix 2 .

The following technical lemma leads to a lower bound for the discrepancy of $\mathcal{D B}_{\text {max }}(n)$.

Lemma 5.2 A maximum weight de Bruijn sequence of order $n$ and maximum weight $d$ has $\left(\begin{array}{c}n-1 \\ d\end{array}\right)$ more Os than $1 s$.

Proof. By definition, a maximum weight de Bruijn sequence of order $n$ and maximum weight $d$ contains every binary string of length $n$ with weight at most $d$ as a substring exactly once. Since each bit in this sequence belongs to $n$ different strings the total number of $1 \mathrm{~s}$ in the sequence is

$$
\begin{aligned}
\text { ones } & =\frac{1}{n} \sum_{j=0}^{d} j\left(\begin{array}{l}
n \\
j
\end{array}\right) \\
& =\frac{0}{n}\left(\begin{array}{l}
n \\
0
\end{array}\right)+\frac{1}{n}\left(\begin{array}{l}
n \\
1
\end{array}\right)+\frac{2}{n}\left(\begin{array}{l}
n \\
2
\end{array}\right)+\cdots+\frac{d}{n}\left(\begin{array}{l}
n \\
d
\end{array}\right) \\
& =0+\left(\begin{array}{c}
n-1 \\
0
\end{array}\right)+\left(\begin{array}{c}
n-1 \\
1
\end{array}\right)+\cdots+\left(\begin{array}{l}
n-1 \\
d-1
\end{array}\right),
\end{aligned}
$$

and the total number of $0 \mathrm{~s}$ is

$$
\begin{aligned}
\text { zeros } & =\frac{1}{n} \sum_{j=0}^{d}(n-j)\left(\begin{array}{l}
n \\
j
\end{array}\right) \\
& =\frac{n}{n}\left(\begin{array}{l}
n \\
0
\end{array}\right)+\frac{n-1}{n}\left(\begin{array}{l}
n \\
1
\end{array}\right)+\frac{n-2}{n}\left(\begin{array}{l}
n \\
2
\end{array}\right)+\cdots+\frac{n-d}{n}\left(\begin{array}{l}
n \\
d
\end{array}\right) \\
& =\left(\begin{array}{c}
n-1 \\
0
\end{array}\right)+\left(\begin{array}{c}
n-1 \\
1
\end{array}\right)+\left(\begin{array}{c}
n-1 \\
2
\end{array}\right)+\cdots+\left(\begin{array}{c}
n-1 \\
d
\end{array}\right) .
\end{aligned}
$$

Thus zeros - ones $=\left(\begin{array}{c}n-1 \\ d\end{array}\right)$.

\footnotetext{
${ }^{2}$ It is also available at http: / / debruijnsequence.org
} 
Theorem 5.3 The de Bruijn sequence $\mathcal{D} \mathcal{B}_{\max }(n)$ has discrepancy at least $\left(\begin{array}{c}n-1 \\ \lfloor n / 2\rfloor\end{array}\right)+\left\lfloor\frac{n}{2}\right\rfloor$.

Proof. Let $d=\lfloor n / 2\rfloor+1$ and $d^{\prime}=\lceil n / 2\rceil$. Recall that $\overline{\mathcal{D}}_{d}(n)$ is a maximum weight de Bruijn sequence with maximum weight $n-d$. Thus, by Lemma 5.2, it has $\left(\begin{array}{c}n-1 \\ n-d\end{array}\right)=\left(\begin{array}{c}n-1 \\ n-(\lfloor n / 2\rfloor+1)\end{array}\right)=\left(\begin{array}{c}n-1 \\ \lfloor n / 2\rfloor\end{array}\right)$ more 0s than 1s. Consider $\overline{\mathcal{D}}_{d}(n)$ with its prefix of $1^{n-d}$ removed. The resulting string, which is a substring of $\mathcal{D} \mathcal{B}_{\text {max }}(n)$, has $\left(\begin{array}{c}n-1 \\ \lfloor n / 2\rfloor\end{array}\right)+(n-d)$ more 0s than 1s. When $n$ is odd we have $n-d=n-\lfloor n / 2\rfloor-1=\left\lfloor\frac{n}{2}\right\rfloor$ and thus $\mathcal{D B}_{\text {max }}(n)$ has discrepancy at least $\left(\begin{array}{c}n-1 \\ \lfloor n / 2\rfloor\end{array}\right)+\left\lfloor\frac{n}{2}\right\rfloor$. When $n$ is even, we additionally add the length $n-1$ prefix of $\mathcal{D}_{d^{\prime}}^{r}(n)$ which has more 0 s than 1 s (exactly one more). Since $n-d+1=n-(\lfloor n / 2\rfloor-1)+1=\left\lfloor\frac{n}{2}\right\rfloor$ (when $n$ is even) this again means that $\mathcal{D} \mathcal{B}_{\text {max }}(n)$ has discrepancy at least $\left(\begin{array}{c}n-1 \\ \lfloor n / 2\rfloor\end{array}\right)+\left\lfloor\frac{n}{2}\right\rfloor$.

By applying Stirling's approximation to $\left(\begin{array}{c}n-1 \\ {[n / 2]}\end{array}\right)$ we obtain the following corollary.

Corollary 5.4 The discrepancy of the de Bruijn sequence $\mathcal{D B}_{\text {max }}(n)$ attains the asymptotic upper bound of $\Theta\left(\frac{2^{n}}{\sqrt{n}}\right)$.

Observe from Table 1 that the discrepancy of $\mathcal{D B}_{\text {max }}(n)$ is exactly $\left(\begin{array}{c}n-1 \\ \lfloor n / 2\rfloor\end{array}\right)+\left\lfloor\frac{n}{2}\right\rfloor$ for $10 \leq n \leq 25$. This leads to the following conjecture.

Conjecture 5.5 The de Bruijn sequence $\mathcal{D B}_{\text {max }}(n)$ has discrepancy equal to $\left(\begin{array}{c}n-1 \\ \lfloor n / 2\rfloor\end{array}\right)+\left\lfloor\frac{n}{2}\right\rfloor$, and moreover, it is the maximum possible discrepancy over all de Bruijn sequences of order $n$.

As noted earlier, the discrepancy of the Cool-lex construction matches the discrepancy for the Weightrange construction for $10 \leq n \leq 25$, except for when $n \bmod 4 \equiv 1$ (see Table 1). As illustration, the Cool-lex construction first constructs cycles of the following weights for $n=6,7,8,9$ :

- $n=6:(0,1,2),(3,4),(5,6)$

- $n=7:(0,1),(2,3),(4,5),(6,7)$

- $n=8:(0,1,2),(3,4),(5,6),(7,8)$

- $n=9:(0,1),(2,3),(4,5),(6,7),(8,9)$

before joining them together one at a time. Note when $n=9$, strings with weights 4 and 5 are grouped together before the smaller cycles are joined together. This causes a reduction in the discrepancy compared to the Weight-range construction. It is possible, however, to tweak the Cool-lex implementation so the discrepancies are equivalent. For instance for $n=9$, the smaller cycles with weights $(0,1,2),(3,4),(5,6),(7,8,9)$ could be joined together instead.

Recently, a shift-rule construction based on the PSR and CSR has been discovered to generate the same sequence as Cool-lex [30]. A discussion on generating de Bruijn sequences applying the PSR and CSR is also given in [12]; it describes joining small cycles together in the same manner as Cool-lex. Thus, we anticipate the resulting sequences would obtain a similar discrepancy profile.

\section{Group 5: LFSR constructions based on primitive polynomials}

In this section we consider de Bruijn sequences that can be generated for a specific $n$ by a primitive polynomial of degree $n$. As discussed by Golomb [20], a primitive polynomial of the form $g(x)=c_{0}+c_{1} x+c_{2} x^{2}+\cdots+c_{n} x^{n}$ over GF(2) corresponds to a feedback function of the form $f\left(a_{1} a_{2} \cdots a_{n}\right)=c_{n} a_{1} \oplus c_{n-1} a_{2} \oplus \cdots \oplus c_{1} a_{n}$. 
Example 2 The primitive polynomial $1+x^{2}+x^{5}$ of degree 5 over GF(2) corresponds to the feedback function $f\left(a_{1} a_{2} a_{3} a_{4} a_{5}\right)=a_{1}+a_{4}$. If $a_{1} a_{2} a_{3} a_{4} a_{5}$ is initialized to 00001, then the LFSR with this feedback function produces the $\mathrm{m}$-sequence

\section{1}

of length $2^{5}-1=31$ when outputting the value $a_{1}$ before each application of the LFSR. By prepending a 0 to the beginning of this $\mathrm{m}$-sequence we obtain a de Bruijn sequence.

To obtain the data in Table 2, we generated all primitive polynomials of degree $n$ for $n=10,11, \ldots, 25$ along with their corresponding LFSRs. The algorithm used to exhaustively list the primitive polynomials is based on the work in [5] and is available at http://debruijnsequence.org/lfsr. We seeded the LFSRs with $0^{n-1} 1$ as described in the above example to obtain a de Bruijn sequence. We then computed the discrepancy of all such sequences. The number of primitive polynomials (and hence LFSRs) of degree $n$ is given by sequence A011260 in the On-Line Encyclopedia of Integer Sequences [1]. This number is listed in the final column of Table 2 .

Below is a list of feedback functions for $n=10,11, \ldots, 25$ that generated de Bruin sequences with discrepancy closest to the corresponding entry for a random sequence.

\begin{tabular}{|c|c|c|c|}
\hline$n$ & Feedback function & Random & Discrepancy \\
\hline 10 & $a_{1} \oplus a_{2} \oplus a_{6} \oplus a_{9}$ & 50 & 46 \\
\hline 11 & $a_{1} \oplus a_{6} \oplus a_{7} \oplus a_{10}$ & 71 & 68 \\
\hline 12 & $a_{1} \oplus a_{4} \oplus a_{7} \oplus a_{8} \oplus a_{9} \oplus a_{12}$ & 101 & 99 \\
\hline 13 & $a_{1} \oplus a_{2} \oplus a_{4} \oplus a_{5} \oplus a_{6} \oplus a_{7} \oplus a_{8} \oplus a_{10} \oplus a_{11} \oplus a_{12}$ & 143 & 143 \\
\hline 14 & $a_{1} \oplus a_{2} \oplus a_{4} \oplus a_{5} \oplus a_{7} \oplus a_{8} \oplus a_{9} \oplus a_{13}$ & 203 & 203 \\
\hline 15 & $a_{1} \oplus a_{2} \oplus a_{6} \oplus a_{11}$ & 288 & 287 \\
\hline 16 & $a_{1} \oplus a_{2} \oplus a_{3} \oplus a_{4} \oplus a_{13} \oplus a_{15}$ & 407 & 406 \\
\hline 17 & $a_{1} \oplus a_{3} \oplus a_{5} \oplus a_{8} \oplus a_{9} \oplus a_{10} \oplus a_{11} \oplus a_{12} \oplus a_{13} \oplus a_{15} \oplus a_{16} \oplus a_{17}$ & 575 & 575 \\
\hline 18 & $a_{1} \oplus a_{4} \oplus a_{5} \oplus a_{7} \oplus a_{10} \oplus a_{11} \oplus a_{12} \oplus a_{15} \oplus a_{16} \oplus a_{17}$ & 815 & 814 \\
\hline 19 & $a_{1} \oplus a_{2} \oplus a_{3} \oplus a_{5} \oplus a_{6} \oplus a_{10} \oplus a_{11} \oplus a_{14} \oplus a_{16} \oplus a_{17}$ & 1157 & 1157 \\
\hline 20 & $a_{1} \oplus a_{2} \oplus a_{3} \oplus a_{4} \oplus a_{5} \oplus a_{6} \oplus a_{7} \oplus a_{8} \oplus a_{9} \oplus a_{10} \oplus a_{11} \oplus a_{12} \oplus a_{13} \oplus a_{15} \oplus a_{16} \oplus a_{17} \oplus a_{19} \oplus a_{20}$ & 1634 & 1633 \\
\hline 21 & $a_{1} \oplus a_{2} \oplus a_{3} \oplus a_{5} \oplus a_{8} \oplus a_{11} \oplus a_{16} \oplus a_{19} \oplus a_{20} \oplus a_{21}$ & 2311 & 2311 \\
\hline 22 & $a_{1} \oplus a_{3} \oplus a_{6} \oplus a_{12} \oplus a_{13} \oplus a_{15} \oplus a_{17} \oplus a_{18} \oplus a_{19} \oplus a_{20} \oplus a_{21} \oplus a_{22}$ & 3264 & 3264 \\
\hline 23 & $a_{1} \oplus a_{2} \oplus a_{3} \oplus a_{4} \oplus a_{5} \oplus a_{6} \oplus a_{9} \oplus a_{10} \oplus a_{12} \oplus a_{14} \oplus a_{16} \oplus a_{17} \oplus a_{18} \oplus a_{23}$ & 4565 & 4565 \\
\hline 24 & $a_{1} \oplus a_{2} \oplus a_{4} \oplus a_{6} \oplus a_{12} \oplus a_{13} \oplus a_{14} \oplus a_{15} \oplus a_{16} \oplus a_{17} \oplus a_{18} \oplus a_{20} \oplus a_{21} \oplus a_{24}$ & 6252 & 6252 \\
\hline 25 & $a_{1} \oplus a_{8} \oplus a_{9} \oplus a_{11} \oplus a_{12} \oplus a_{16} \oplus a_{20} \oplus a_{21} \oplus a_{24} \oplus a_{25}$ & 9192 & 9192 \\
\hline
\end{tabular}

\section{Future directions and open problems}

In this paper, we investigated the discrepancies of 13 de Bruijn sequence constructions. We proved that two constructions attain the lower bound of $\Theta(n)$ and presented one new construction that attains the upper bound of $\Theta\left(\frac{2^{n}}{\sqrt{n}}\right)$. It remains an interesting problem to demonstrate a generic construction for all $n$ with discrepancy that is close to that of a random stream of bits of the same length. Some additional avenues of future research include the following.

1. Simplify the description of the Huang construction [22]. Does it have the smallest discrepancy over all de Bruijn sequences? 
2. Answer the conjectures regarding the discrepancies for the greedy Pref-same and Pref-opposite constructions (Conjecture 3.1 and Conjecture 3.2).

3. Analyze the discrepancy of PCR4 which had discrepancy closest to one we might expect from a random stream of bits.

4. Determine whether or not the maximum discrepancy of any de Bruijn sequence is $\left(\begin{array}{c}n-1 \\ \lfloor n / 2\rfloor\end{array}\right)+\left\lfloor\frac{n}{2}\right\rfloor$ (Conjecture 5.5).

5. Generalize the investigation of discrepancy to de Bruijn sequences over an arbitrary alphabet size $k$.

6. Study the distribution of discrepancy over all possible de Bruijn sequences.

\section{Acknowledgement}

The research of Joe Sawada is supported by the Natural Sciences and Engineering Research Council of Canada (NSERC) grant RGPIN-2018-04211.

\section{References}

[1] OEIS Foundation Inc. (2020), The On-Line Encyclopedia of Integer Sequences, http://oeis.org.

[2] A. Alhakim. A simple combinatorial algorithm for de Bruijn sequences. The American Mathematical Monthly, 117(8):728-732, 2010.

[3] A. Alhakim, E. Sala, and J. Sawada. Revisiting the Prefer-same and Prefer-opposite de Bruijn sequence constructions. Theoretical Computer Science, 852:73-77, 2021.

[4] S. R. Blackburn and I. E. Shparlinski. Character sums and nonlinear recurrence sequences. Discrete Math., 306(12):1126-1131, June 2006.

[5] K. Cattell, F. Ruskey, J. Sawada, M. Serra, and C. Miers. Fast algorithms to generate necklaces, unlabeled necklaces, and irreducible polynomials over GF(2). Journal of Algorithms, 37(2):267-282, 2000.

[6] J. Cooper and C. Heitsch. The discrepancy of the lex-least de Bruijn sequence. Discrete Mathematics, 310:1152-1159, 2010.

[7] J. Cooper and C. E. Heitsch. Generalized Fibonacci recurrences and the lex-least de Bruijn sequence. Advances in Applied Mathematics, 50:465-473, 2010.

[8] P. B. Dragon, O. I. Hernandez, J. Sawada, A. Williams, and D. Wong. Constructing de Bruijn sequences with co-lexicographic order: The $k$-ary Grandmama sequence. European Journal of Combinatorics, 72:1-11, 2018.

[9] P. B. Dragon, O. I. Hernandez, and A. Williams. The grandmama de Bruijn sequence for binary strings. In Proceedings of LATIN 2016: Theoretical Informatics: 12th Latin American Symposium, Ensenada, Mexico, pages 347-361. Springer Berlin Heidelberg, 2016.

[10] C. Eldert, H. Gray, H. Gurk, and M. Rubinoff. Shifting counters. AIEE Trans., 77:70-74, 1958. 
[11] P. L. Emerson and R. D. Tobias. Computer program for quasi-random stimulus sequences with equal transition frequencies. Behavior Research Methods, Instruments, \& Computers, 27(1):88-98, Mar 1995.

[12] T. Etzion. Self-dual sequences. Journal of Combinatorial Theory, Series A, 44(2):288 - 298, 1987.

[13] H. Fredricksen. A survey of full length nonlinear shift register cycle algorithms. SIAM Review, 24(2):195-221, 1982.

[14] H. Fredricksen and I. Kessler. Lexicographic compositions and de Bruijn sequences. J. Combin. Theory Ser. A, 22(1):17 - 30, 1977.

[15] H. Fredricksen and J. Maiorana. Necklaces of beads in $k$ colors and $k$-ary de Bruijn sequences. Discrete Math., 23:207-210, 1978.

[16] D. Gabric and J. Sawada. A de Bruijn sequence construction by concatenating cycles of the complemented cycling register. In Combinatorics on Words - 11th International Conference, WORDS 2017, Montréal, QC, Canada, September 11-15, 2017, Proceedings, pages 49-58, 2017.

[17] D. Gabric and J. Sawada. Constructing de Bruijn sequences by concatenating smaller universal cycles. Theoretical Computer Science, 743:12-22, 2018.

[18] D. Gabric, J. Sawada, A. Williams, and D. Wong. A framework for constructing de Bruijn sequences via simple successor rules. Discrete Mathematics, 241(11):2977-2987, 2018.

[19] S. Golomb. On the classification of balanced binary sequences of period $2^{n}-1$ (corresp.). IEEE Transactions on Information Theory, 26(6):730-732, November 1980.

[20] S. W. Golomb. Shift Register Sequences. World Scientific, Singapore, 2017.

[21] Y. Hsieh, H. Sohn, and D. Bricker. Generating $(n, 2)$ de Bruijn sequences with some balance and uniformity properties. Ars Combinatoria, 72:277-286, 072004.

[22] Y. Huang. A new algorithm for the generation of binary de Bruijn sequences. J. Algorithms, 11(1):4451, 1990.

[23] C. J. A. Jansen, W. G. Franx, and D. E. Boekee. An efficient algorithm for the generation of DeBruijn cycles. IEEE Transactions on Information Theory, 37(5):1475-1478, Sep 1991.

[24] M. H. Martin. A problem in arrangements. Bull. Amer. Math. Soc., 40(12):859-864, 1934.

[25] J. Massey. Shift-register synthesis and $\mathrm{BCH}$ decoding. IEEE Transactions on Information Theory, 15(1):122-127, January 1969.

[26] A. A. Philippakis, A. M. Qureshi, M. F. Berger, and M. L. Bulyk. Design of compact, universal DNA microarrays for protein binding microarray experiments. In T. Speed and H. Huang, editors, Research in Computational Molecular Biology, pages 430-443, Berlin, Heidelberg, 2007. Springer Berlin Heidelberg.

[27] A. Ralston. A new memoryless algorithm for de Bruijn sequences. J. Algorithms, 2(1):50-62, 1981.

[28] F. Ruskey, J. Sawada, and A. Williams. De Bruijn sequences for fixed-weight binary strings. SIAM Journal on Discrete Mathematics, 26(2):605-617, 2012. 
[29] E. Sala, J. Sawada, and A. Alhakim. Efficient constructions of the Prefer-same and Prefer-opposite de Bruijn sequences. CoRR, abs/2010.07960, 2020.

[30] J. Sawada and A. Williams. Universal cycles for strings with fixed content based on cool-lex order. manuscript, 2021.

[31] J. Sawada, A. Williams, and D. Wong. Universal cycles for weight-range binary strings. In Combinatorial Algorithms - 24th International Workshop, IWOCA 2013, Rouen, France, July 10-12, 2013, LNCS 8288, pages 388-401, 2013.

[32] J. Sawada, A. Williams, and D. Wong. The lexicographically smallest universal cycle for binary strings with minimum specified weight. Journal of Discrete Algorithms, 28:31-40, 2014.

[33] J. Sawada, A. Williams, and D. Wong. A surprisingly simple de Bruijn sequence construction. Discrete Math., 339:127-131, 2016.

[34] H.-S. Sohn, D. L. Bricker, J. R. Simon, and Y. Hsieh. Optimal sequences of trials for balancing practice and repetition effects. Behavior Research Methods, Instruments, \& Computers, 29(4):574-581, Dec 1997. 


\section{A Table of discrepancies}

\begin{tabular}{c|cccc|ccc} 
& \multicolumn{7}{|c}{ ( Group 1 ) } \\
$n$ & Huang & CCR2 & CCR3 & CCR1 & Pref-same & $\begin{array}{c}\text { ( Group 2 ) } \\
\text { Lexp }\end{array}$ & Pref-opposite \\
\hline 10 & 12 & 13 & 13 & 16 & 24 & 24 & 27 \\
11 & 13 & 14 & 15 & 18 & 29 & 29 & 34 \\
12 & 15 & 16 & 16 & 22 & 35 & 35 & 43 \\
13 & 16 & 17 & 18 & 23 & 43 & 43 & 52 \\
14 & 18 & 19 & 20 & 30 & 48 & 48 & 63 \\
15 & 19 & 21 & 21 & 29 & 59 & 59 & 74 \\
16 & 21 & 22 & 23 & 36 & 68 & 68 & 87 \\
17 & 22 & 24 & 25 & 37 & 79 & 79 & 100 \\
18 & 24 & 26 & 26 & 43 & 88 & 88 & 115 \\
19 & 25 & 27 & 28 & 43 & 103 & 103 & 130 \\
20 & 27 & 29 & 30 & 52 & 114 & 114 & 147 \\
21 & 28 & 31 & 31 & 50 & 127 & 127 & 164 \\
22 & 30 & 32 & 33 & 59 & 142 & 142 & 183 \\
23 & 31 & 34 & 35 & 59 & 155 & 155 & 202 \\
24 & 33 & 36 & 36 & 67 & 172 & 172 & 223 \\
25 & 35 & 37 & 38 & 66 & 187 & 187 & 244 \\
26 & 36 & 39 & 40 & 77 & 208 & 208 & 267 \\
27 & 38 & 41 & 42 & 74 & 224 & 224 & 290 \\
28 & 40 & 43 & 43 & 85 & 246 & 246 & 315 \\
29 & 41 & 44 & 45 & 84 & 264 & 264 & 340 \\
30 & 43 & 46 & 47 & 94 & 286 & 286 & 367 \\
& & & & & & &
\end{tabular}

\begin{tabular}{|c|c|c|c|c|c|c|c|}
\hline \multirow[b]{2}{*}{$n$} & \multicolumn{5}{|c|}{ ( Group 3 ) } & \multicolumn{2}{|c|}{ ( Group 4 ) } \\
\hline & PCR4 & Random & PCR3 & PCR2 & Prefer-1/PCR1 & Cool-lex & Weight-range \\
\hline 10 & 29 & 50 & 75 & 101 & 120 & 131 & 131 \\
\hline 11 & 41 & 71 & 141 & 180 & 222 & 257 & 257 \\
\hline 12 & 51 & 101 & 248 & 321 & 416 & 468 & 468 \\
\hline 13 & 70 & 143 & 468 & 587 & 784 & 801 & 930 \\
\hline 14 & 85 & 203 & 850 & 1065 & 1488 & 1723 & 1723 \\
\hline 15 & 110 & 288 & 1604 & 1974 & 2824 & 3439 & 3439 \\
\hline 16 & 175 & 407 & 2965 & 3632 & 5376 & 6443 & 6443 \\
\hline 17 & 246 & 575 & 5594 & 6785 & 10229 & 11452 & 12878 \\
\hline 18 & 326 & 815 & 10461 & 12635 & 19484 & 24319 & 24319 \\
\hline 19 & 462 & 1157 & 19765 & 23746 & 37107 & 48629 & 48629 \\
\hline 20 & 730 & 1634 & 37243 & 44585 & 71250 & 92388 & 92388 \\
\hline 21 & 954 & 2311 & 70575 & 84270 & 138332 & 167975 & 184766 \\
\hline 22 & 1327 & 3264 & 133737 & 159281 & 268582 & 352727 & 352727 \\
\hline 23 & 1820 & 4565 & 254322 & 302449 & 521553 & 705443 & 705443 \\
\hline 24 & 2684 & 6252 & 484172 & 574819 & 1012795 & 1352090 & 1352090 \\
\hline 25 & 3183 & 9192 & 924071 & 1096009 & 1966813 & 2496163 & 2704168 \\
\hline 26 & 4108 & 13074 & 1766284 & 2092284 & 3819605 & 5200313 & 5200313 \\
\hline 27 & 5604 & 17933 & 3382851 & 4004050 & 7453523 & 10400613 & 10400613 \\
\hline 28 & 7629 & 22672 & 6488970 & 7672443 & 14544826 & 20058314 & 20058314 \\
\hline 29 & 10433 & 34591 & 12468181 & 14730243 & 28382864 & 37442182 & 40116614 \\
\hline 30 & 13637 & 57357 & 23991972 & 28316271 & 55421919 & 77558775 & 77558775 \\
\hline
\end{tabular}




\section{B C program to construct maximum-discrepancy de Bruijn sequences}

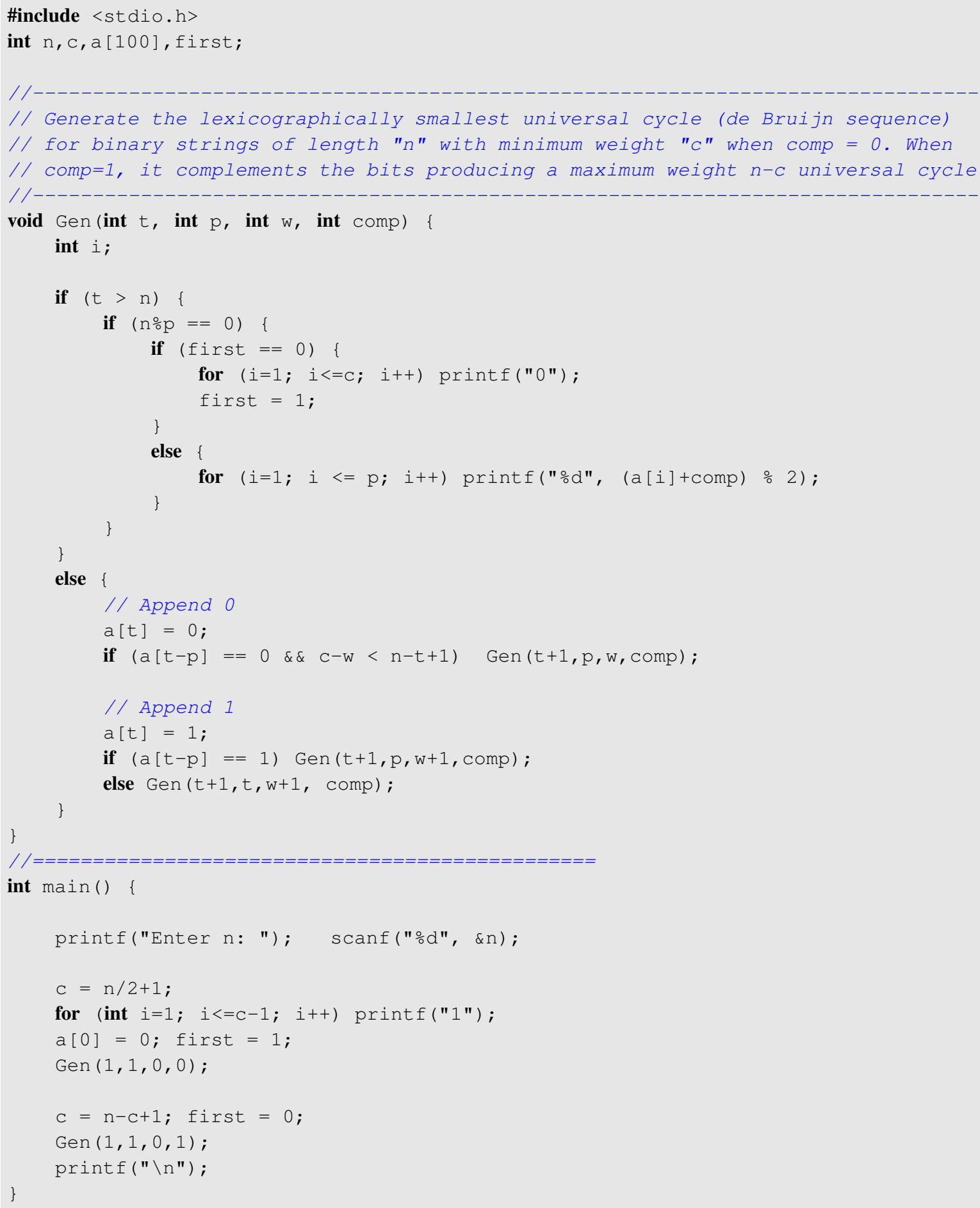

